\title{
Pros and Cons of Low-kV Transmission Electron Microscopy
}

\author{
R.F. Egerton \\ Physics Department, University of Alberta, Edmonton, Canada T6G 2E1
}

For many years, higher resolution in the TEM meant increasing the accelerating voltage but with the advent of lens-aberration correctors, the following advantages of lower voltages $(<100 \mathrm{kV})$ have been recognized.

Reduction in knock-on damage, which disappears below some threshold voltage that is typically above $200 \mathrm{kV}$ for bulk displacement [1] but can be below $100 \mathrm{kV}$ for surface displacement, such as sputtering into the vacuum [2] or displacement along a surface [3].

Increased image contrast, due to an increase in elastic and inelastic scattering within the specimen. This advantage disappears if the specimen is thick enough that plural or multiple scattering dominates.

More localized inelastic scattering: the delocalization distance decreases by about a factor of two between $200 \mathrm{kV}$ and $30 \mathrm{kV}$ [4] - of importance in low-loss EELS, where the delocalization distance can exceed 1 nm. Also reduced Cerenkov loss, which simplifies measurement of local bandgap by EELS [5]. In addition, lower $\mathrm{kV}$ makes it easier to obtain good energy resolution and to analyze volume losses characteristic of the interior of the specimen, since the bulk-loss intensity is proportional to $1 / v^{2}$ whereas surface losses scale according to $1 / v, v$ being the incident-electron speed.

However, the following disadvantages of low-kV operation are apparent.

Need for very thin specimens, particularly if the atomic number is high. This requirement is easily satisfied for some materials (e.g. graphene, nanotubes) but it can in crease the difficulty of specimen preparation techniques or result in the observed properties being dominated by surface oxide or contamination layers.

Increased electrostatic charging of insulating specimens, which can deflect the incident electron beam and give rise to micro-lensing or ionic motion [6] or dielectric breakdown

[7] within the specimen.

Reduced electron-optical resolution, due to increased aberration of the electron lenses, although this factor becomes less important when a spherical-aberration corrector is incorporated in the TEM [8]. Chromatic aberration becomes severe below $50 \mathrm{kV}$, requiring a Cc-corrector or (for high-resolution STEM) a monochromator. 
In any event, electron-optical resolution is largely irrelevant in the case of beam-sensitive (e.g. organic) specimens, where ionization damage limits the dose-limited resolution $\delta$ :

$$
\delta=(\mathrm{SNR})(\mathrm{DQE})^{-1 / 2}(\mathrm{FDc} / \mathrm{e})^{-1 / 2}(2+\mathrm{C})^{-1 / 2} / \mathrm{C}(1)
$$

This resolution depends on the image contrast $\mathrm{C}$, the signal-collection efficiency $\mathrm{F}$ and the characteristic dose Dc that the specimen can withstand; $\delta$ improves with increasing specimen thickness until nonlinear effects (e.g. plural scattering) become important; see Fig.1. In the case of a very thin specimen $(\mathrm{C}<<2)$, the resolution for bright-field scattering contrast is better at lower $\mathrm{kV}$ (despite a decrease in Dc) because the scattering power and contrast are higher (Fig.1a). For phase-contrast imaging, $\delta$ is independent of $\mathrm{kV}$ because decrease in Dc is compensated by an increase in phase shift, until the latter reaches some limiting value; see Fig. 1b.

\section{References:}

[1] ML Jenkins and MA Kirk, Characterization of Radiation Damage by TEM (Institute of Physics, 2001).

[2] RF Egerton et al., Ultramicroscopy 110 (2010), p. 991.

[3] RF Egerton, Microscopy and Microanalysis 19 (2013), in press.

[4] RF Egerton, Ultramicroscopy 107 (2007) p.575. .

[5] M Stoeger-Pollach, Micron 39 (2008), p. 1092.

[6] N Jiang and J Silcox, J. Appl. Phys. 92 (2002), p. 2310.

[7] J Cazaux, Ultramicroscopy 60 (1995), p. 411.

[8] P Hawkes (ed.) Aberration-corrected Electron Microscopy, (Academic Press, Amsterdam). (a) (b)
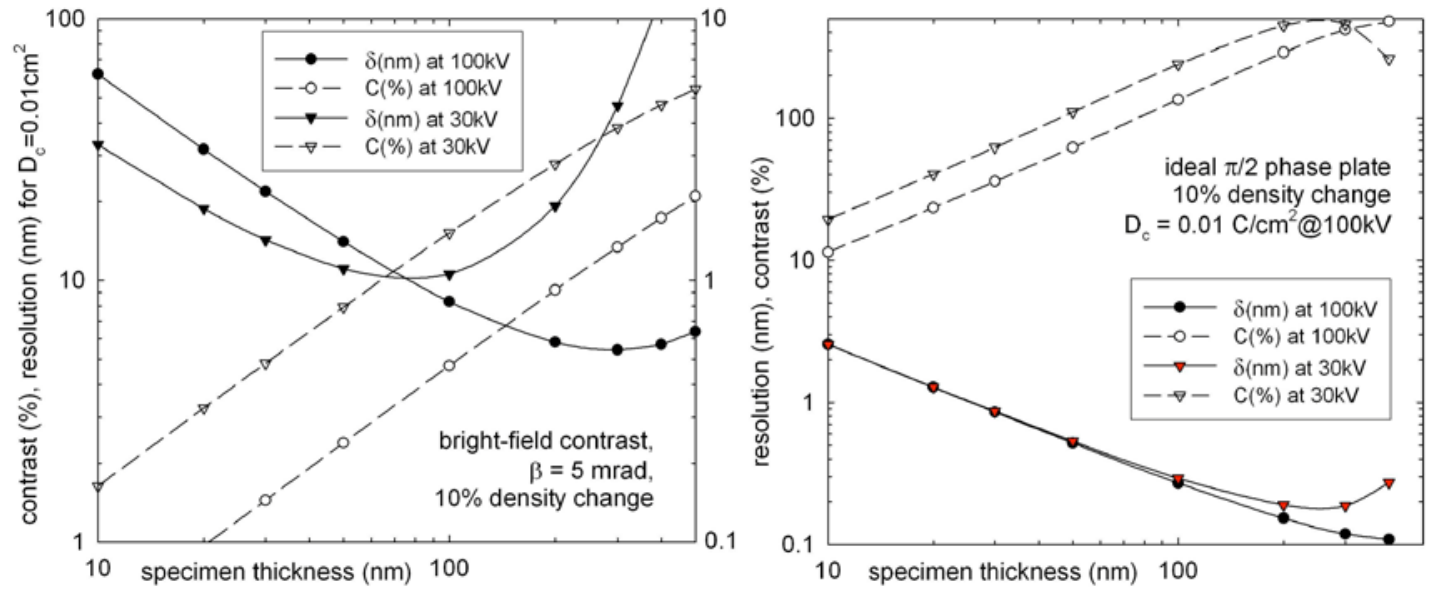

Figure 1. Dose-limited resolution $\delta$ and contrast $\mathrm{C}$ at a boundary with a $10 \%$ density change within an amorphous polymer having $\mathrm{Dc}=0.01 \mathrm{C} / \mathrm{cm}^{2}$, calculated from Eq.(1) for (a) bright-field scattering contrast with a $5 \mathrm{mrad}$ objective aperture, and (b) phase contrast with an ideal $\pi / 2$ phase plate (no absorption) at the specimen back-focal plane. 